\title{
Identification of the Air Supply System for Combustion, With the Help of Artificial Neural Networks
}

\author{
Enrique Santana Lopez ${ }^{1}$, Deynier Montero Góngora*2 and Orlando Víctor Vega Arias ${ }^{3}$ \\ ${ }^{1}$ Department of Higher Metallurgical Mining Institute of Moa "DrC Antonio Núñez Jiménez", Cuba \\ ${ }^{2}$ University of Camagüey "Ignacio Agramonte Loynaz", Cuba \\ ${ }^{3}$ Production Company of Nickel and Cobalt "Comandante Ernesto Che Guevara", Cuba
}

Received: 眥 October 03, 2018; Published: 䟧 October 23, 2018

*Corresponding author: Deynier Montero Góngora, Department of Higher Metallurgical Mining Institute of Moa DrC Antonio Núñez Jiménez, Cuba

\begin{abstract}
The production of nickel in Cuba is one of the main export items in our economy. In recent years, its production costs have risen significantly, with a high incidence of electricity costs, which is why it is necessary to take energy shock measures to reverse this situation. Currently there are deficiencies in the Reduction Furnace plant related to the control of the air supply and the electric power used by the asynchronous motors that drive the centrifugal fans, reducing the efficiency levels of the production process and the plant in general. In order to increase the energy efficiency of the combustion process supply system and reach an optimum control model of the airflow of this plant, variants are designed and simulated based on artificial neural networks that allow to establish the air demand from the drive of the fans by means of variable speed drives.
\end{abstract}

Keywords: Model; Air flow; Speed Variators

\section{Introduction}

The Mining and Metallurgical Industry has become one of the bases on which the economic-industrial development of the country is based and is one of the ones that currently faces the challenge of Business Improvement, a way to achieve a global competitive level. This entrepreneurial improvement as an integral process cannot avoid the technological improvement based on a consistent application of advances in science and technology. In the nickeliferous plants in which the carbonate-ammonia leaching technology is used, also universally known as the "Caron" process, the multi-sole hearth furnaces, which represent an important link in the chain, are used in the process of mineral reduction. productive The Reduction Furnaces plant has a total of nine fans driven by $220 \mathrm{~kW}$ engines, of which six remain in permanent operation and in the previous works it has been shown that there are reservations in the consumption of electric power that exceed 15\%. In [1] and [2] the inefficiency of the current control of the combustion air system used by this plant and the present energy reserves is demonstrated, since manually the excess air flow is regulated, while the fans work at full capacity all the time which produces an unnecessary expenditure of electricity. The amount of energy lost during one year due to the regulation of the damper, only in one fan, is approximately 150MWh. In [3] the state of the supply network and the insufficiency in the operation of the fans are shown to achieve the pressure required by the system. Also in [4], the experimental identification of the post-combustion sub-process of a multihousehold reduction furnace was carried out, through a process of trial and error, an artificial neuron network of the multilayer perceptron type was obtained, able to predict the temperature of the four home with an error range of - 8 to $5 \%$ and of - 4 to $1 \%$ for home temperature six. The present investigation has like objective the experimental identification of the system of provision of air by means of the utilization of artificial neural networks "feedforward", for the future implementation of a control architecture with view to increase the energetic efficiency of this process. It has been shown that neural networks can be used effectively and accurately for the identification and control of systems with complex dynamics, especially for nonlinear plants that vary in time and that are more difficult to regulate with conventional methods [5]. In particular, feedforward neural networks have been the most widely used for these purposes. The growing interest in neural networks is due to 
its great versatility and the continuous advance in network training algorithms and hardware [6]. As software to carry out the research, the MATLAB program was used.

\section{Content}

\section{The Process of Reduction}

The Herreshoff furnaces [7] are composed of a metallic cylinder lined internally with refractory bricks, protected externally by a metal casing, facilities for agitation, feeding and discharging the ore and combustion chambers. They are formed internally by 17 hearths or soleras that have the shape of spherical vaults. They also have a rotating central shaft to which 68 arms are articulated, four for each hearth (Figure 1). After the ore is weighed, it is discharged to the auger feeder which transports the mineral to the zero hearth in the upper part of the kiln, being retained inside for approximately
45 minutes. The reduction process is eminently endothermic. For this reason, the furnace has 10 combustion chambers placed in homes $15,12,10$, eight and six with high pressure oil burners, which are responsible for producing hot gases for heating the ore, at the same time that enrich the reducing atmosphere of the furnace since they work with incomplete combustion. Thus, the ore in its descent is subjected to a rising temperature profile that allows a gradual heating, while entering a gaseous profile that guarantees an increase in the concentration of reducers. The chemical reagent in the process is the mixture of carbon monoxide and hydrogen, so the concentration of these gases in contact with minerals, at the beginning of the decomposition is extremely important. After reducing the nickel oxide and the higher oxides of iron to metallic nickel and metallic iron respectively, the mineral mixture is discharged from the furnace to a rotating conveyor.

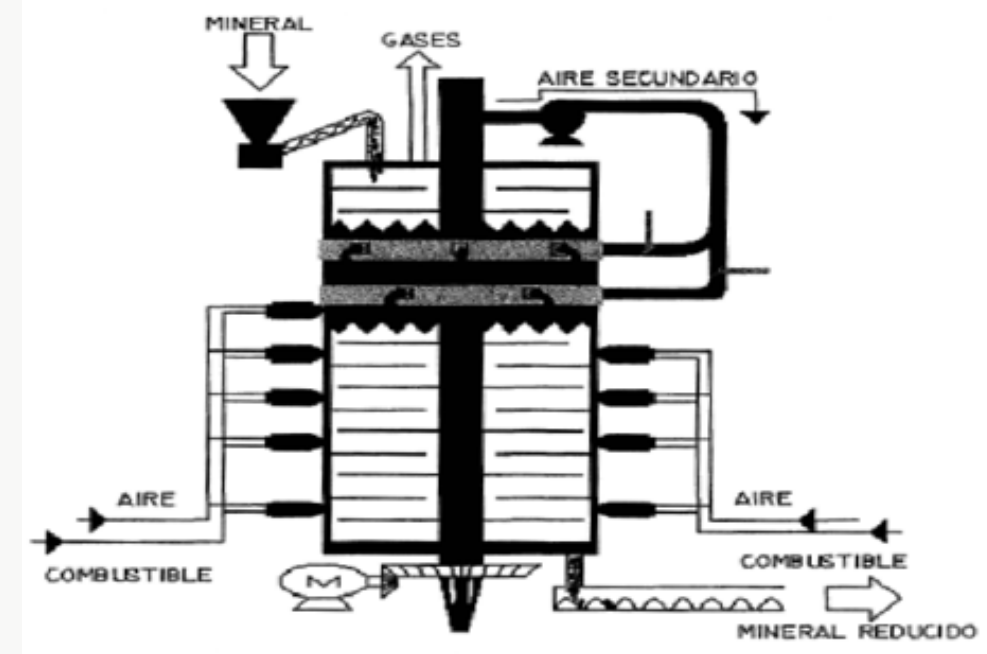

Figure 1: Reduction Furnace.

\section{Description of the Combustion Air System}

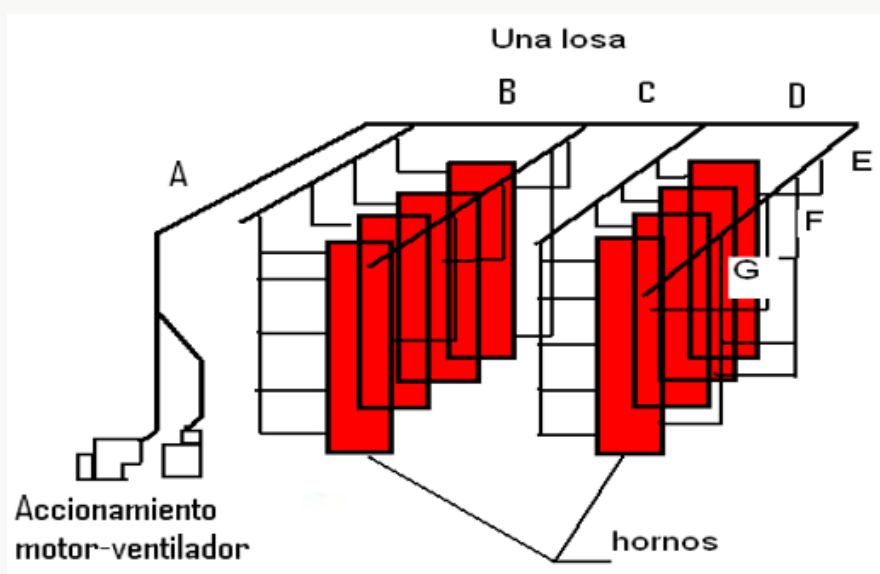

Figure 2: Composition of a slab of the kilns plant.

In the "Furnaces of Reduction" plant there are nine delta connection three-phase induction motors, of which three are reserve. These engines are working in an underloaded regime, which have a high starting torque and after the current is restored do not operate at its rated power, the motors are connected by four belts to its centrifugal forced draft fan (suction), which represent the 
mechanical load for the asynchronous machine. The fans generate a flow of air that is supplied to the combustion chambers (PRIOR), which mix the oil and air, then goes to the oven. Each furnace needs at least six cameras to carry out the process [8]. In the plant, a slab is the structure of three motor-fan drives, with one of reserve and the network of pipes that correspond to the connection with the eight ovens with their relevant cameras (Figure 2).

\section{Obtaining an Approximate Model of the Process Through Experimental Identification}

The obtaining of a mathematical model that describes the process from the phenomenological point of view would be extremely complicated, since the physical-chemical processes involved would lead to differential equations systems in partial derivatives, nonlinear and variable in time. Therefore, experimental identification was chosen [4].

\section{Notions of Modeling Using Artificial Neural Networks}

Neural networks provide in the identification of some systems more precise models than with conventional methods, especially for non-linear systems with variable parameters [6].The determination of the type of artificial neural network, the number of layers and the number of neurons in each layer that best characterize the air supply process for the combustion chambers was carried out through a trial and error process in the which is played with the number of neurons and the maximum admissible error. Through Matlab's Toolbox (nnstart), different types of transfer and training functions were tested. For the performance of artificial neural models, the mean square error and the correlation coefficient were used between the real values and those obtained by the network following the recommendations of [9]. The objective was to provide

Table 1: Summary of the experiments carried out. the network with an adequate number of neurons in the hidden layer, to learn the characteristics of the possible relationships between the sample data. The variables considered in the artificial neural model were linearly scaled between 0 and 1 .

\section{Analysis of the Experiments Carried Out}

In the investigated process, the speeds of the motors manipulated by three variable speed drives were taken as input variables; that drive the centrifugal fans seven, eight and nine of slab three and as output variable the air pressure in the collector. Several experiments were performed alternating the operation of the fans similar to what was shown in [3] and positive and negative steps were applied at different points of operation to the input variables, with a sampling period of three seconds taking into account the dynamics of the process see Table 1 . The air pressure in the collector ranged between 8.8 and $15.4 \mathrm{kPa}$ of all the configurations tested for the operation of the fans, the best results were obtained according to the performance of the process (air pressure in the collector of $14 \mathrm{kPa}$ ) that of working with fans seven and eight, in a range of operation for both from 97 to $100 \%$ in the engine speed, with the nine reserve fan. The active powers consumed by the motors of fans seven and eight were from 196 to $205 \mathrm{~kW}$. The behavior of the correlation coefficients for the training, validation, testing and adjustment of the artificial neural network identified are shown in Figure 3. As a result of processing 12198 data from a set of experiments ("Output" is assumed as the air pressure in the collector calculated by the artificial neural model and "Target" the actual air pressure in the collector). Figure 4 shows the generalization of the network with 40476 data not presented during the training, where a correlation coefficient of 0.71 is observe

\begin{tabular}{|c|c|c|c|c|c|}
\hline Sl.No & $\begin{array}{c}\text { Number of } \\
\text { Combustion Chambers }\end{array}$ & Manipulated Variables & $\begin{array}{c}\text { Levels of The Manipulated } \\
\text { Variables (\%) }\end{array}$ & Constant Variables & $\begin{array}{c}\text { Levels of the Constant } \\
\text { Variable (\%) }\end{array}$ \\
\hline 1 & 49 & rpmV8 & $90-100$ & rpmV9 & 100 \\
\hline 2 & 49 & rpmV9 & $95-100$ & rpmV8 & 85 \\
\hline 3 & 49 & rpmV8 & $87-93$ & rpmV9 & 100 \\
\hline 4 & 49 & rpmV9 & $89-100$ & rpmV8 & 89 \\
\hline 5 & 49 & rpmV8 & $90-93$ & rpmV9 & 98 \\
\hline 6 & 49 & rpmV7 & $97-99$ & rpmV8 & 97 \\
\hline 7 & 49 & rpmV7 & $90-91$ & rpmV8 & 90 \\
\hline 8 & 49 & rpmV8 & $93-94$ & rpmV7 & 94 \\
\hline \multirow{2}{*}{9} & \multirow{2}{*}{49} & \multirow{2}{*}{ rpmV7 } & \multirow{2}{*}{$70-80$} & rpmV8 & 90 \\
\hline & & & & rpmV9 & 100 \\
\hline \multirow{2}{*}{10} & \multirow{2}{*}{49} & \multirow{2}{*}{ rpmV8 } & \multirow{2}{*}{$85-90$} & rpmV7 & 90 \\
\hline & & & & rpmV9 & 95 \\
\hline \multirow{2}{*}{11} & \multirow{2}{*}{49} & \multirow{2}{*}{ rpmV9 } & \multirow{2}{*}{$80-85$} & rpmV7 & 85 \\
\hline & & & & rpmV8 & 85 \\
\hline
\end{tabular}



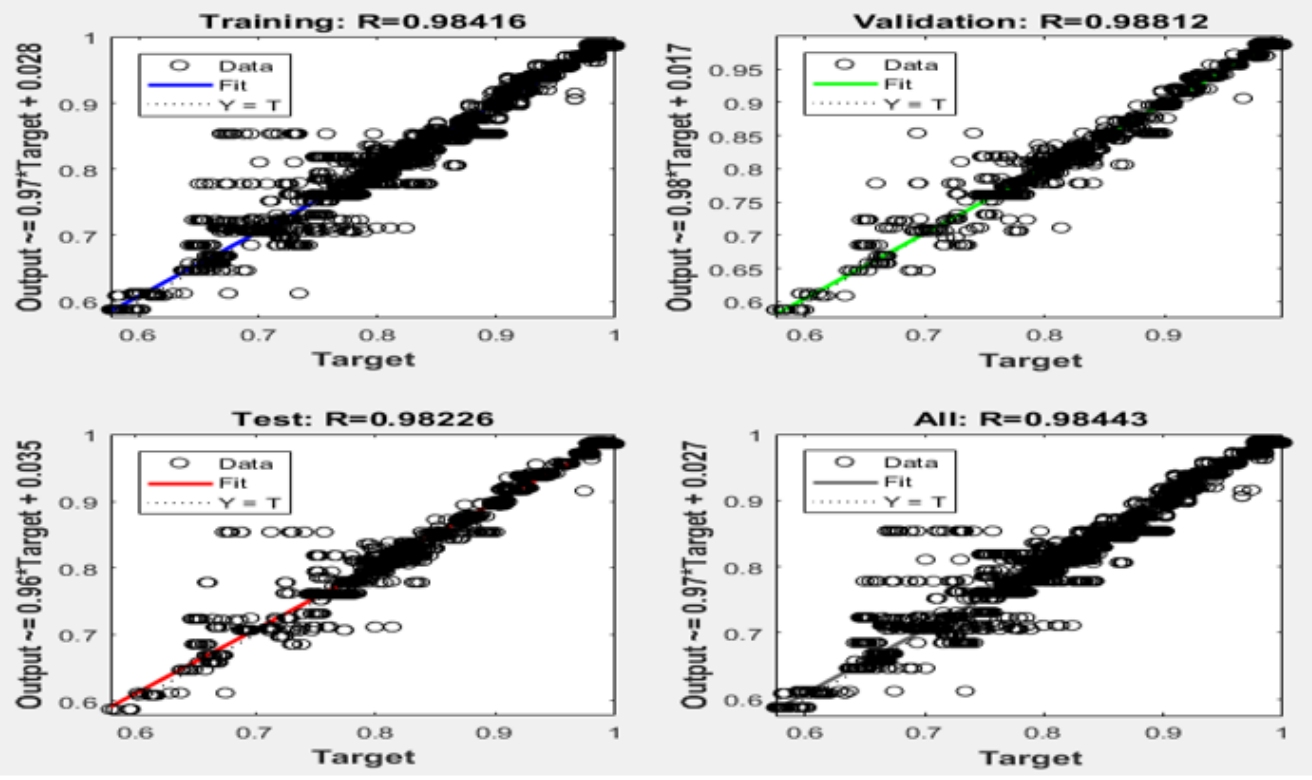

Figure 3: Correlation coefficients of the neural network.

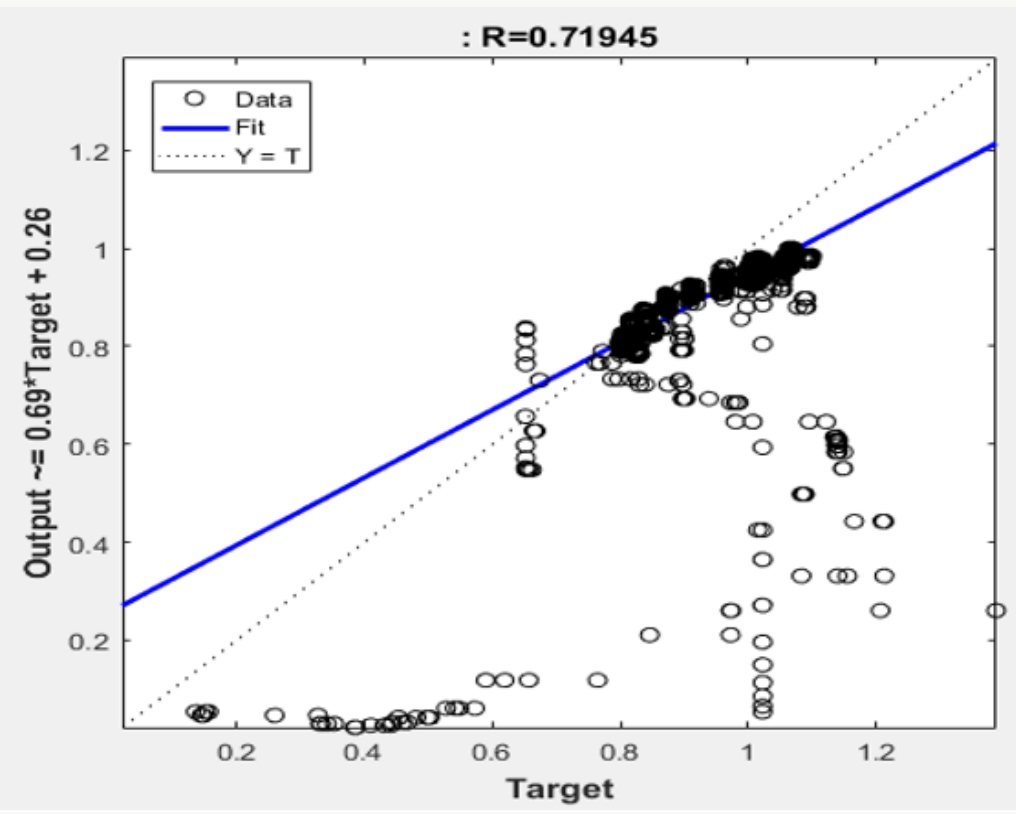

Figure 4: Generalization of the network.

\section{Conclusion}

With the operation of two fans (seven and eight) in a range of operation for both of 97 to $100 \%$ in the speed of the motor, keeping the fan nine of reserve; the best results were obtained according to the benefits of the process. For the identification of the air supply system for combustion; The artificial neural network of the feedforward-backpropagation type, constituted by 10 neurons in the hidden layer and one in the output layer, with logsigmoidal and linear activation functions respectively, with the LevenbergMarquardt training algorithm, has been selected for its ability to approximate non-linear functions. The artificial neural model proposed has been able to predict the air pressure in the collector with a $98 \%$ correlation and $71 \%$ in its generalization.

\section{References}

1. Martínez E (2011) Speed regulation in Atmospheric Air Fans of combustion of the Reduction Furnaces plant. Electrical Moa: Undergraduate Thesis.

2. Santana, E, Marrero S Control of combustion air flow in reduction furnaces. University of Camagüey. 2013. International Conference CYTDES "Ciencia y Tecnología por una desarrollo sostenible.

3. Vega $O$ (2015) Evaluation of the operational parameters of the combustion air supply in the Reduction Furnaces. Moa: Master's Thesis. 
4. Montero D (2016) Modeling the post-combustion in a multi-household furnace using artificial neural networks. ISMM Master's thesis.

5. Valverde R, Gachet D (2007) Identification of dynamic systems using RBF neural networks. RIAI, 4: 32-42.

6. Santos M (2011) An Applied Approach to Intelligent Control. RIAI 8: 283-296.

7. Habashi F (1997) Handbook of Extractive Metallurgy. s.l. : Wiley-VCH, Weinheim (2).
8. (2007) Operations Manual of the Basic Production Unit of the Reduction Furnace Plant. Company Comandante Ernesto Che Guevara.

9. Sutradhar, A, Sengupta, A, Challa R (2010) Annual IEEE India Conference. Department of Electrical Engineering, Bengal Engineering \& Science University. Identification of Servo-driven Inverted Pendulum System using Neural Network.
(C) Commons Attribution 4.0 License

To Submit Your Article Click Here: Submit Article

DOI: $10.32474 /$ ARME.2018.01.000113

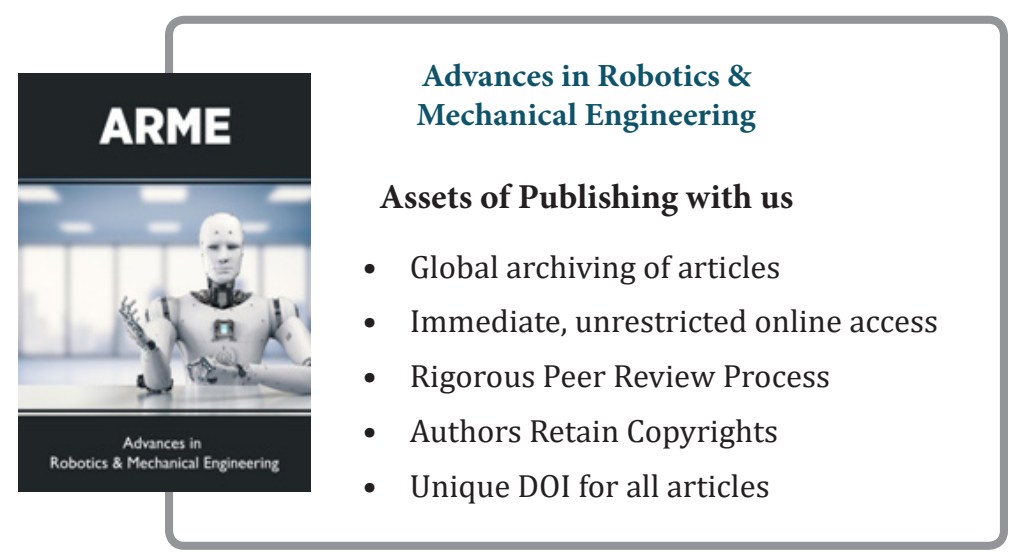

